\title{
Dynamical evolution of objects on highly elliptical orbits near high-order resonance zones
}

\author{
Eduard D. Kuznetsov and Stanislav O. Kudryavtsev
}

\author{
Kourovskaya Astronomical Observatory, Ural Federal University, \\ Lenin ave., 51, Ekaterinburg, 620000 Russia \\ email: eduard.kuznetsov@urfu.ru
}

\begin{abstract}
Both analytical and numerical results are used to study high-order resonance regions in the vicinity of Molnya-type orbits. Based on data of numerical simulations, long-term orbital evolution are studied for objects in highly elliptical orbits depending on their area-to-mass ratio. The Poynting-Robertson effect causes a secular decrease in the semi-major axis of a spherically symmetrical satellite. Under the Poynting-Robertson effect, objects pass through the regions of high-order resonances. The Poynting-Robertson effect and secular perturbations of the semimajor axis lead to the formation of weak stochastic trajectories.
\end{abstract}

Keywords. celestial mechanics, highly elliptical orbit, high-order resonance, satellite, stochastic trajectory

\section{Introduction}

Region of highly elliptical orbits (HEO) has a very complex dynamics. Both active and passive objects are moved on HEO. There is a problem of protecting active satellites from space debris. It requires high-accuracy propagation of HEO objects motion. These objects have a long-term evolution of eccentricities and inclinations due to the LidovKozai resonance (Lidov (1962) and Kozai (1962)). There are secular perturbations of semi-major axes due to the atmospheric drag. The Poynting-Robertson effect also leads to secular perturbations of semi-major axes for objects with area-to-mass ratio (AMR) more than $1 \mathrm{~m}^{2} / \mathrm{kg}$ (Kuznetsov et al. (2012)). The dynamical evolution of high AMR objects in the vicinity of Molniya-type orbits was studied by Sun et al. (2013). In this paper, a vicinity of Molniya orbit is considered. There are several high-order resonance zones in this region. A stochastic trajectory formation due to objects passage through high-order resonance zones was considered.

\section{High-order resonances}

Frequencies of perturbations caused by the effect of sectoral and tesseral harmonics of the Earth's gravitational potential are linear combinations of a satellite's mean motion $n_{M}$, angular velocities of pericenter motion $n_{g}$ and node motion $n_{\Omega}$ of it's orbit, and angular velocity of the Earth $\omega$.

Following Allan (1967a) and (1967b), we form the frequencies

$\nu_{1}=p\left(n_{M}+n_{\Omega}+n_{g}\right)-q \omega, \quad \nu_{2}=p\left(n_{M}+n_{g}\right)+q\left(n_{\Omega}-\omega\right), \quad \nu_{3}=p n_{M}+q\left(n_{g}+n_{\Omega}-\omega\right)$, where $p, q$ are integers. 
The condition $\nu_{1} \approx 0$ corresponds to $p: q$ resonance between the satellite's mean motion $n_{M}$ and the Earth's angular velocity $\omega$. This condition represents $n$-resonance. The condition $\nu_{2} \approx 0$ corresponds to $i$-resonance under which a position of the ascending node of an orbit repeats periodically in a rotating coordinate system. The condition $\nu_{3} \approx 0$ corresponds to $e$-resonance at which a position of the line of apsides is considered.

We estimated the values of semi-major axis corresponding to the $n$-, $i$ - and $e$-resonances from the conditions $\nu_{1}=0, \nu_{2}=0$, and $\nu_{3}=0$ in the vicinity of the Molniya orbit. Initial conditions corresponded to HEO with the eccentricity 0.65 and the critical inclination $63.4^{\circ}$. Semi-major axis values varied from $26000 \mathrm{~km}$ to $27100 \mathrm{~km}$. There were $17 \mathrm{high}-$ order resonance relations $p: q$ between the mean motion of angular orbital elements and the Earth's angular velocity: $16 \leqslant|p| \leqslant 25,33 \leqslant|q| \leqslant 49$. The orders of resonance are $49 \leqslant|p|+|q| \leqslant 74$.

\section{Stochastic trajectory formation}

To conduct of the long-term orbital evolution research, we used "A Numerical Model of Artificial Satellite Earth Motion" created by our colleagues from the Tomsk State University Research Institute of Applied Mathematics and Mechanics (Bordovitsyna et al. (2007)). The model of disturbing forces accounted for the nonsphericity of gravitational field of the Earth (model EGM96, harmonics up to the $27^{\text {th }}$ order and degree inclusive), the attraction of the Moon and the Sun, the tides in the Earth's body, the direct radiation pressure, taking into account the shadow of the Earth (the reflection coefficient of the satellite surface $k=1.44$ ), the Poynting-Robertson effect, and the atmospheric drag. The model utilized Everhart's method of the $19^{\text {th }}$ order.

The initial conditions corresponded to HEO with the eccentricity $e_{0}=0.65$ and critical inclination $i_{0}=63.4^{\circ}$. Initial semi-major axes $a_{0}$ values were consistent with resonant conditions arisen from the analytical approximation. AMRs tried were equal to 0.02, 0.2, and $2 \mathrm{~m}^{2} / \mathrm{kg}$. The time span for simulation was 24 years.

The Poynting-Robertson effect causes a secular decrease in the semi-major axis of a spherically symmetrical satellite. Secular decrease in the semi-major axis, for a satellite with $\mathrm{AMR}=2 \mathrm{~m}^{2} / \mathrm{kg}$ near Molniya orbit, equals approximately $0.5 \mathrm{~km} /$ year. The effect weakens slightly, in resonance regions. Under the Poynting-Robertson effect, objects pass through high-order resonances regions. The Poynting-Robertson effect and secular perturbations of semi-major axis lead to the formation of weak stochastic trajectories.

\section{Acknowledgement}

Authors would like to thank Mrs. Anna Glazyrina for reading and correcting the paper.

This work was supported by the Russian Foundation for Basic Researches (grant 1302-00026a).

\section{References}

Allan, R. R. 1967, Planet. Space Sci., 15, 53

Allan, R. R. 1967, Planet. Space Sci., 15, 1829

Bordovitsyna, T. V., Baturin, A. P., Avdyushev, V. A., \& Kulikova, P. V. 2007, Izv. Vyssh. Uchebn. Zaved., Fiz., 50, 60

Kozai, Y. 1962, AJ, 67, 591

Kuznetsov, E. D., Zakharova, P. E., Glamazda, D. V., Shagabutdinov, A. I., \& Kudryavtsev, S. O. 2012, Solar System Research, 46, 442

Lidov, M. L. 1962, Planet. Space Sci., 9, 719

Sun, R.-y., Zhao, C.-y., Zhang, M.-j., \& Hou, Y.-G. 2013, Adv. Sp. Res., 51, 2136 